\title{
PSICOLOGIA DA ERA VIRTUAL: Atitudes de estudantes adolescentes frente ao 0 rkut
}

\section{Psychology of virtual age: A dolescent students' attitudes toward 0 rk ut}

\author{
Diogo C. S. Ferreiraa, Carlos E. Pimentel ${ }^{b}$, Carlos da Silva Cirinoc, \\ Helder Silva Santos ${ }^{\mathrm{d}}$, Maitê Costa de Oliveira ${ }^{\mathrm{e}}$
}

\footnotetext{
a D outor em Ciências do Comportamento pelaUniversidade de Brasília(UnB), Professor Assistente I do Instituto de Educação Superior de Brasília (IE SB), Brasília, D F - Brasil, e-mail: diogo_seco@ yahoo.com.br

b D outorando em Psicologia Social na Universidade de Brasília (ŪnB), Mestre em Psicologia Social pela Universidade Federal da Paraíba(UFPB), Brasília, D F - Brasil, e-mail:kdu1976@ gmail.com

c Professor A djunto III de Psicologia da Universidade Tiradentes (UNIT), Aracaju, SE - Brasil.

d Aluno do curso de G raduação em Psicologia da UNIT, Aracaju, SE - Brasil.

e Aluna do curso de G raduação em Psicologia da UNIT, A racaju, SE - Brasil.
}

\begin{abstract}
Resumo
0 presente estudo consistiu em conhecer quais as principais vantagens e desvantagens da participação dos jovens no site de relacionamento social O rkut, propor uma escala para medir atitudes frente ao O rkut e conhecer a relação destas atitudes com variáveis sócio-demográficas. Isso pode interessar à psicologia social, da mídia ou psicologia da internet. Para a realização deste propósito levou-se a cabo uma pesquisa com 444 estudantes ( $m=17$ anos, $d p=2,52)$, da cidade de A racaju (SE). Foi utilizado um procedimento-padrão para coleta de dados através de um questionário composto por questões sobre vantagens e desvantagens do O rkut, atitudes gerais frente ao O rkut e questões sócio-demográficas. 0 s resultados principais mostram que a principal vantagem da participação do O rkut é "fazer amigos" e a principal desvantagem reportada foi a "falta de privacidade". A análise psicométrica demonstrou que as atitudes frente ao site podem ser aferidas de modo válido, preciso e breve, independente de sexo, série e classe socioeconômica.
\end{abstract}

Palavras-chave: A titudes; O rkut; Site de relacionamento social. 


\begin{abstract}
The purpose of the present study consisted on knowing the main advantages and disadvantages of the participation of youngsters on the Orkut social network website and then to propose a scale to measure the attitudes towards this site, as well as to understand the relations of these attitudes to social demographics variables. These findings will be of interest to social psychologists, media psychologists or internet psychologists. To attain these objectives, a research with $444(m=17$ years old, $d p=2,52)$ high school students from the city of Aracaju was conducted. A standard procedure to obtain the data was utilized, with a questionnaire composed of questions concerning the advantages and disadvantages of the Orkut, general attitudes towards the site and social demographics questions. The results show that the main advantage of orkut affiliation is "friendship" and the main disadvantage reported was "lack of privacy". The psychometric analysis indicated that the attitudes towards the site could be measured in a valid, precise and brief way, independently of gender, grade level and socio economic status.
\end{abstract}

Keywords: Attitudes; Orkut; Social network website.

\section{INTRODUÇÃO}

A internet com seus diversos recursos - $e$ mails, chats, blogs e sites de relacionamento social - tem gerado novas formas de interação, influenciando 0 pensamento, sentimento e comportamento e pode ser considerada um laboratório psicológico (Skitka \& Sargis, 2006). De acordo com o IBGE (2007), a Pesquisa Nacional por Amostra de Domiclílios PNAD de 2005 sobre acesso à internet e posse de telefone móvel celular para uso pessoal, verificou que 32,1 milhões de pessoas acessaram a internet em 2005, sendo, entre estas, 13,9 milhões de estudantes.

$O$ levantamento realizado pelo IBGE, em parceria com o Comitê G estor da Internet no Brasil - CGI.br, mostrou, também, que os internautas tinham, em média, 28 anos de idade, mas o maior índice está na faixa entre 15 e 17 anos (33,9\%) e de 10 a 14 anos, com 24,4\% deles acessando ainternet. Desse total de acessos, parece que muitos se dedicaram à visita de sites de relacionamento (Social $\mathrm{N}$ etworking Site, SN S). De modo extremamente expressivo, o site de relacionamento Orkut (www.orkut.com), conseguiu alcançar uma parcela importante de usuários. A propósito, Araújo (2006, p. 30) explica que:

O Orkut em menos de um ano, conseguiu ultrapassar a fantástica marca de 2.000.000 membros e, atualmente, deste total, $75 \%$ dos usuários são brasileiros, superando em muito o contingente de usuários americanos que, em números percentuais, equivale a 6,5 $\%$ do total de participantes.

Considerando a prevalência desse fenômeno, essas interações so ciais propiciadas pelo desenvolvimento tecnológico, pelos sites de relacionamento social, devem figurar na agenda de pesquisas dos psicólogos sociais. Com o fim de contribuir com esta área, desenvolveu-se, portanto, uma pesquisa empírica cujo objetivo principal foi conhecer as atitudes de estudantes frente ao site de relacionamento social O rkut. Antes da apresentação do método e resultados da pesquisa empírica, revisou-se rapidamente a área da psicologia da mídia, a cyberpsychology e pesquisas psicológicas sobre websites, objeto de estudo da presente pesquisa.

\section{Psicologia da $\mathrm{m} \backslash$ ídia}

A psicologia da mídia usa conceitos e métodos da psicologia para estudar o impacto dos meios de comunicação de massa em indivíduos, grupos e culturas (Fischoff, 2005). O surgimento da psicologia da mídia pode ser datado a há cerca de mais de 90 anos, com os trabalhos de Hugo Münsterberg, de 1916, o primeiro a verificar a influência do filme na audiência e L.L. Thurstone, em 1928, acerca da mensuração de atitudes frente ao cinema, indicando que os filmes podem influenciar 
negativamente o comportamento dejovens(Fischoff, 2005). Outro importante trabalho nesse início de configuração da área é o de Cantril eAllport, de 1935, sobre a psicologia do rádio (Giles, 2003).

Esse autor ainda explica que o trabalho na psicologia da mídia centrou-se amplamente no uso da televisão por crianças e na influência desta na aquisição de habilidades de leitura, assim como na imitação do comportamento antissocial. A psicologia da mídia é representada pela D ivisão 46 da A merican Psychological A ssociation (APA) e, de acordo com esta associação, faz parte de seu escopo o estudo de aspectos psicológicos da mídia, o que inclui o rádio, televisão, filmes, vídeo, notícias, revistas e últimas tecnologias, fomentando, ainda, pesquisas sobre 0 impacto da mídia no comportamento humano (Media Psychology, 2007). Numa verificação específica dos conteúdos da revista M edia Psychology, podem-se perceber pesquisas atuais na área da influência da TV e internet em variáveis como agressividadee sexualidade, mas, em geral, os estudos na psicologia da mídia ainda persistem em focar principalmente nas formas tradicionais de mídia, como a TV e mídia impressa (ver Giles, 2003).

Por outro lado, ainda podem ser encontrados relatos de pesquisas sobre a influência de vídeo games violentos enão violentos e a respeito da influência de letras de música no comportamento sexual. Com efeito, Giles (2003) explica que a psicologia da mídia tem um amplo escopo que envolve a psicologia da propaganda, a psicologia da música e a psicologia da internet além de trabalhos fora da psicologia, nas ciências da comunicação e pesquisas sobre a mídia. Ademais, os estudos da mídia podem ser importantes para 0 desenvolvimento da psicologia cognitiva, psicologia do desenvolvimento e psicologia social. Por outro lado, apsicologia da mídia podeabordar seus tópicos de estudo a partir de diversas perspectivas como a cognitiva e social, e a Escola de Frankfurt e a Sociologia Norte-Americana são as duas maiores influências na teorização sobre a mídia (Giles, 2003). 0 presente estudo opta pela perspectiva sócio-cognitiva, preponderante na psicologia social contemporânea (Rodrigues, Assmar \& Jablonski, 2000; Smith \& Mackie, 1995; Zajonc, 1968).

Luskin (2003) explica que, na psicologia da mídia, ainda se encontra a pscybermedia, neologismo que representa a psicologia, inteligência artificial (cibernética) e a mídia. Giles (2003) destaca que a pesquisa sobre vários aspectos da Internet tem crescido nos últimos 10 a 20 anos e que, na psicologia, a cyberpsychology é uma área que vem estudando as implicações da tecnologia virtual para o corpo, identidade e natureza humana em geral. Portanto, verifica-se que esta é uma subárea de pesquisas na psicologia da mídia.

\section{Psicologia da era virtual: A cyberpsicologia e o uso da Internet}

No livro seminal de 1999, The Psychology of Internet, Patrícia Wallace estabelece as bases para umavariedadedepesquisaspsicológicas concernentes à interação pela internet, como formação de impressões, role playing, dinâmica de grupos, agressividade, atratividade, pornografia, adicção, altruísmo e gênero (Wallace, 1999). D esde então, uma série de periódicos tem dedicado suas páginas à divulgação de pesquisas que tem a interação pela internet como seu objeto de estudos, como Behavior and Information T echnology, Computers in $\mathrm{H}$ uman Behaviors, Cyberpsychology and Behavior e o International Journal of H uman-C omputer Studies. Não há, no entanto, consenso sobre a definição da área, seus objetos de estudo ou mesmo suas futuras direções. Muitos trabalhos aparecem como extensões de pesquisas em psicologia social, investigando a adequação de determinados conceitos como autoimagem, personalidade e anonimidade no novo cenário da internet (McKenna \& Bargh, 2000), enquanto outros sugerem uma adequação mais cuidadosa desses conceitos à luz de uma nova epistemologia (Teske, 2002). Há, ainda, aqueles direcionados à internet como um meio pelo qual se pode fazer a pesquisa (experimental, survey) psicológica (Birnbaum, 2000; G osling, Vazire, Srivastava \& John, 2004; Pereira, Silva \& Silva, 2006; Skitka \& Sargis, 2006).

O sprimeirostrabalhosnaáreasedividiram entre visões pessimistas e comentários mais otimistas. Turkle (1995) alertou para o perigo da possibilidade de destruição de integração social significativa cara a cara, sendo substituída por elos muito mais frágeisevoláteis construídos pelainternet e Stoll (1995) acrescenta que as "reais amizades" (aquelas não criadas pela internet) poderiam perder seu valor. Teske (2002) comenta que reações dessa natureza são frequentes e a comunidade científica já se posicionou assim frente a outras inovações tecnológicas anunciadas como destruidoras das relações sociais como nós as conhecemos, como 0 
rádio e a televisão. Em uma posição mais otimista, Rheingold (1993) afirma que a internet poderia trazer benefícios para 0 relacionamento social, possibilitando relacionamentos sem restrições por localização geográfica e suavizando o efeito dos principais "gargalos" de início de relacionamento como aparência, etnia, gênero, nível educacional e socioeconômico. Com isso, haveria um maior pluralismo, diversidade e liberdade individuais nos relacionamentos sociais. Somente com 0 aparecimento de pesquisas empíricas, no final do século XX, que posições mais ponderadas surgiram.

0 primeiro trabalho metodologicamente rigoroso a investigar o efeito do uso da internet sobre os relacionamentos sociais foi conduzido por Kraut e colaboradores, com 93 domicílios estadunidenses, publicado em 1998 (Kraut, et al., 1998). Os autores obtiveram informações sobre características pessoais que poderiam influenciar no uso da internet, bem como realizaram um acompanhamento da amostra por dois anos subsequentes à coleta de dados. Seus resultados levaram os autores a cunhar o conceito de "Paradoxo da Internet", pois uma tecnologia criada para comunicação interpessoal pode ter como resultado a diminuição de envolvimento social, bemestar psicológico e envolvimento emocional e substituir relacionamentos mais ricos por contatos pessoais superficiais. Os autores concluíram que 0 uso frequente da internet esteve relacionado com a diminuição da comunicação familiar, participantes mais extrovertidos e com maiores redes sociais anteriores ao início do estudo usaram a internet menos, o uso da internet esteve associado a uma diminuição dos círculos sociais locais, solidão e depressão iniciaisnão predisseram uso maisfrequente da internet e os adolescentes são os maiores usuários da internet para relacionamento social.

McKenna e Bargh (2000) pedem cautela na análise dos resultados desse estudo. Além de algumas limitações metodológicas, como a falta de um grupo de controle e a representatividade da amostra, esses autores afirmam que os efeitos observados podem variar com diferenças individuais e diferenças nos objetivos e necessidades no uso da internet. Embora não haja ainda nenhuma conclusão sobre o efeito dainternet nas comunicações interpessoais, existem claras indicações de que a anonimidade, o papel menor desempenhado pela aparência física e distância geográfica, e a possibilidade de maior controle do ritmo e andamento das interações desempenham um papel significativo nas diferenças entre relacionamentos face a face e via internet. Essas características tendem a diminuir a modéstia dos participantes (Tice, Butler, Muraven \& Stillwell, 1995), aumentar a tendência a apresentar selves idealizados (McKenna \& Bargh, 2000), facilitar a descoberta de familiaridades e similaridades importantes para a atração interpessoal (Byrne, 1971) e evitar os efeitos negativos de sinais não verbais que acompanham as relações face a face, como expressões de cansaço e tédio.

Com isso, o estudo da psicologia do uso da internet ainda é um campo aberto a novas pesquisas. Características dos relacionamentos virtuais, como anonimidade, facilidade de acesso, falta de privacidade e ênfase na linguagem verbal merecem investigações mais detalhadas, assim como características de personalidade que podem estar relacionadas ao maior uso dessa modalidade de relacionamento, em detrimento de relacionamentos face a face. Com o surgimento de redes sociais com milhões de usuários espalhados por todo 0 mundo além do Orkut (como MySpace e Facebook), é especialmente importante a descoberta das características desta nova forma de relacionamento via websites e seus impactos nos relacionamentos humanos presenciais previamente estabelecidos, com familiares e amigos próximos.

\section{Websites Pesquisas prévias na psicologia}

Uma das formas de se estudar websites é através das atitudes frente a estes (Chen \& Wells, 1999).Asatitudes têm sido utilizadas para seinvestigar uma enormidade de questões. G ordon W. Allport, na década de 30, já havia assinalado que esse se trata de um dos conceitos mais distintivos e indispensáveis de toda a psicologia social contemporânea (Allport, 1966). Apesar de mais de meio século da afirmação allportiana, o construto de atitude continua tendo foco destacável não só na psicologia, mas também nas ciências sociais (Ajzen, 2001). Ajzen (2001) explica que as atitudes são avaliações que podem se expressar em dimensões como bom/ ruim, benéfico/ prejudicial, prazeroso/ desprazeroso. As atitudes podem ser conceituadas, portanto, como avaliações gerais sobre diversos objetos sociais (Crites, Fabrigar \& Petty, 1994), como as drogas, um partido político ou uma instituição, como a Igreja, ou mesmo, como no presente caso, um website. 
$\mathrm{Wu}$ (1999) conceitua as atitudes frente a websites como sendo derivadas de três classes de informação: a) cognitiva; b) afetiva e c) comportamental, ou ainda como a quantidade de afetos positivos ou negativos frente a websites. Chen e Wells (1999) definem estas como sendo predisposições de web surfers para responder de modo favorável ou desfavorável frente ao conteúdo da web em situações naturais e verificaram que atitudes favoráveis frente a websites se relacionam positivamente com informação $(r=0,68)$, entretenimento $(r=0,51)$ e organização $(r=0,44)$. Diversas pesquisas sobre atitudes frente a websites são realizadas na área da psicologia do consumidor. McMillan, Hwang e Lee (2003), por exemplo, realizaram uma pesquisa sobre quatro sites de hotéis com 311 consumidores, verificando que as atitudes positivas eram mais associadas àqueles sites em que se podiam fazer reservas online. Outros têm se interessado em verificar características diversas de websites que se relacionam com atitudes de compras online (Shergill \& Chen, 2003). Lee, Hong e Lee (2004), por sua vez, verificaram que atitudes favoráveis de estudantes universitários em relação a websites predizem o comportamento de consumo de marcas e a fidelidade à marca e que 0 conhecimento do produto moderou essa relação.

Com relação a sites de relacionamento especificamente, não se tem verificado muitas pesquisas. Isso deve se explicar em parte por se tratar de um fenômeno recente, do início deste século (Ellison, Steinfield \& Lampe, 2007; O liveira, 2007; Pierce, 2006). Tamyra A. Pierce, da Universidade da Califórnia, Fresno, recentemente apresentou resultados de duas pesquisas na revista online Journal of M edia Psychology (Pierce, 2006; Pierce, 2007) sobre o site de relacionamento MySpace.

Na primeira investigação (Pierce, 2006), a autora verificou que $42 \%$ dos adolescentes participantes afirmaram que seus pais sabem que eles participam do site, mas apenas $26 \%$ dos pais visitam os perfis dos filhos. Ademais, revelou-se que apenas $15 \%$ dos estudantesafirmaram que seus pais discutem "riscos negativos" da utilização da internet. Pierce (2006) explica que os usos e a teoria da satisfação sugerem que os diversos meios de comunicação servem para cumprir necessidades diferentes e que os diferentes sites na internet ou serviços cumpririam necessidades diferentes. "A futura pesquisa deve examinar essa área mais detalhadamente" (Fure, 2006). Na segunda pesquisa (Pierce, 2007), Pierce verificou o conteúdo do MySpace em relação a: 1) informações demográficas; 2) informações pessoais; 3) material visual sexual; 4) profanidade eprofanidade sexual e 5) número de amigos adicionados pelo usuário. D e acordo com resultados dessa pesquisa, a autora conclui que os usuários do MySpace disponibilizam umagrandevariedade deinformações pessoais incluindo conteúdo sexual, profanidade e obscenidade sexual. Verificou, ademais, que os mais jovens colocam mais informações sobre contatos pessoais (telefone, endereço residencial) e que as mulheres postam mais informações pessoais e fotos sexuais (nudez) que os homens.

Ellison, Steinfield e Lampe (2007) recentemente publicaram seus resultados sobre os benefícios do Facebook. Verificando que fatores demográficos, medidas bem-estar subjetivo e certos tipos de uso da internet predizem a quantidade de capital social (recursos adquiridosno curso das relações sociais) reportadapelosestudantes. 0 uso do Facebook e o capital social variaram com base na autoestima e satisfação com a vida. Aqueles estudantes que reportaram baixa satisfação e autoestima parecem ganhar capital social se usam mais (intensidade) 0 Facebook. Os autores sugerem que os recursos dos SNS podem ser particularmente úteis para esses estudantes, que têm dificuldades na socialização, em manter vínculos off-line com pessoas e grupos sociais.

No Brasil, tendo em conta a pesquisa psicológica, encontraram-se apenas duas pesquisas empíricas sobre o Orkut, site de relacionamento social criado em 2004 e que fez mais sucesso aqui no Brasil, contribuindo com a maior parte dos usuários, que no exterior (Araújo, 2006; Oliveira, 2007). A pesquisa se trata da dissertação de mestrado "O rkut: O impacto da realidade da infidelidade virtual" da PUC do Rio de Janeiro de Maria Engel de Oliveira, orientadapelo professorBernardo Jablonski. O liveira (2007) entrevistou pelo msn 10 usuários do Orkut (faixaetária de 18a26anos deidade) queparticipavam de comunidades sobre traição/ infidelidade entre casais, e analisou depoimentos deixados em comunidades. Oliveira (2007) buscou conhecer sentimentos e opiniões dos usuários do Orkut sobre a infidelidade online. De acordo com as respostas desses usuários, a troca de mensagens mais ardentes, carinhosas ou ilícitas, a visualização de fotos de outros homens e mulheres pelo site foi considerada como traição. A outra pesquisa empírica sobre 0 Orkut foi um trabalho de conclusão de curso na mesma universidade supracitada (Hamann, 2004). 
0 interesse em conhecer as atitudes dos adolescentes se explica pela popularidade do site entre esse grupo, apesar das restrições da lei (o site é proibido para menores de 18 anos) e pelo uso acentuado da internet por esse grupo (IBG E , 2007). O s quais, muitas vezes são alvos de crimes sexuais, como pedofilia, e apresenta características que certamente coloca-o em risco com relação a diversos comportamentos ilícitos reforçados nas várias comunidades do Orkut. De acordo com o relator especial da O rganização das Nações Unidas (O NU), Juan Miguel Petit, o problema da pedofilia no cyber espaço é cada vez maior e merece uma legislação específica (ONU Brasil, 2007). Estudar essas crianças e adolescentes é particularmente significativo por conformarem a cyberculture, a "geração virtual" (Giles, 2003), mas nenhuma pesquisa sobreatitudes ante ao $\mathrm{O}$ rkutfoi encontrada em nosso país, nem medida alguma para a mensuração dessas atitudes (considerando-se busca no Google Acadêmico com a expressão "atitudes frente ao Orkut" em 03 de outubro de 2007).

Objetivando ampliar o conhecimento sobre os SN Sa partir da psicologia, preliminarmente buscou-se conhecer as vantagens e desvantagens da participação no Orkut, a direção e magnitude das atitudes de estudantes adolescentes ao usar 0 O rkut, verificando diferenças com relação avariáveis sócio-demográficas, como sexo e idade. Tendo em conta que estudos sobre o O rkut encontrados em nosso país são de índole qualitativa (Hamann, 2004; O liveira, 2007) e a importância de se contar com uma medida autoadministrável (self-report) tipo lápis e papel, busca-se, também, verificar a estrutura fatorial e consistência interna das atitudes frente ao Orkut, propondo uma forma válida (validade de construto), precisa e prática de mensuração dessas atitudes que possa ser uma opção para pesquisas na psicologia da mídia, psicologia social, da adolescência e clínica.

\section{MÉTODO}

\section{Participantes}

Participaram da pesquisa 444 estudantes, com idade média de 17 anos ( $\mathrm{dp}=2,52$, amplitude de 14 a 42 anos) escolhidos de modo não probabilístico, sendo 168 homens (38\%) e 274 mulheres (62\%), de escolas públicas e particulares da cidade de Aracaju (SE, Brasil). 97,5\% destes estudantes são adolescentes e jovens, 108 destes alunos cursavam a primeira série do ensino médio (24,3\%), 122 a segunda (27,5\%) e $213(48 \%)$ faziam a terceira durante o primeiro semestre do ano de 2007. 50,7\% destes eram de escola particular e $49,3 \%$ da rede pública, $51,1 \%$ de classe média e $26,1 \%$ de classe média alta e, com o objetivo de se conhecer a identidade cultural, verificou-se que 78\% desses estudantes residiram mais da metade de suas vidas em Aracaju.

\section{Instrumento}

Escala de A titudes. Crites e colaboradores (1994) elaboraram 4 itens globais ancorados em escalas tipo diferencial semântico de 7 pontos (variando de $-3 \mathrm{a}+3$ ) para mensurar atitudes gerais, no sentido de avaliações gerais. Esses itens foram testados em vários objetos atitudinais (literatura, casamento, perdas financeiras, controle de natalidade, igreja e cobras) com índices de consistência interna (Alfa de Cronbach; á) variando de 0,90 a 0,96 (á médio = 0,95) (Crites et al., 1994). Simons e Carey (2000), por sua vez, verificaram a precisão desses itens, á $=0,97$, para atitudes em relação ao uso de maconha, com a escala de 9 pontos (variando de -4 a +4). Gouveia, Pimentel, Queiroga, Meira e Jesus (2005), no contexto brasileiro, verificam para a versão em português da escala (Simons \& Carey, 2000): uma estrutura unifatorial, com variância total explicada de 84,1\% e índice de consistência interna elevado (á $=0,94)$, além de índices de ajuste bastante animadores: $\div 21$ g.l. $=0,87, \mathrm{GFI}=1, \mathrm{~A} \mathrm{GFI}=0,99$ e RMR $=0,01$. G ouveia, Pimentel, Medeiros, G ouveia e Palmeira (2007) ainda verificaram a validade e precisão dessa medida também no que se refere ao uso de drogas, encontrando valor próprio de 3,17, com $79,3 \%$ da variância total explicada e elevada consistência interna (á $=0,91)$.

No presente estudo utiliza-se essa versão em português (G ouveia et al., 2005) denominandose a medida, portanto, de E scala de A titudes frente ao Orkut(EAFO).O participantedeveiniciararesponder essesitens com base no estímulo: "Considero o sitede relacionamento Orkut..." Abaixo dessa frase estão os quatro pares deadjetivos, itens, (negativo/ positivo, desagradável/ agradável, ruim/ bom e indesejável/ desejável) situados nos extremos do diferencial 
semântico de9pontos. Aspontuações são codificadas para a digitação: 1, 2, 3 e 4 representam atitudes desfavoráveis, sendo 50 ponto nulo, enquanto as pontuações 6, 7, 8 e 9 indicam atitudes favoráveis. No final desta escala foi incluído um questionário sócio-demográfico, que visou coletar dados do participante, como sexo, idade, tipo de escola, série, classe socioeconômica percebida (baixa, média baixa, média, média alta e alta), e horas de uso de internet. Ainda foram incluídas duas questões sobre vantagens e desvantagens da participação no O rkut. Contou-se para essa pesquisa com uma amostra de 10 alunos do último ano do ensino fundamental $\left(9^{\circ}\right.$ ano) para verificar se a EAFO era compreensível (validade semântica). Dada à simplicidade desta, nenhuma alteração foi demandada.

\section{PROCEDIMENTO}

Utilizou-se de um procedimento-padrão para pesquisa de coleta de dados por amostragem. As direções das escolas escolhidas foram visitadas pelos autores deste trabalho com o objetivo de agendar uma aplicação de questionários em sala de aula. Nessa ocasião ainda era explicado o objetivo geral da pesquisa e garantido todos os cuidados do ponto de vista dos procedimentos éticos da pesquisa com seres humanos. Ademais, os pesquisadores se comprometiam em apresentar os resultados em formato de palestras para os alunos e toda a escola. Essas informações foram repetidas quando da coleta nas salas. Os questionários, portanto, foram respondidos de forma coletiva pelos alunos que, em média, levaram 10 minutos para isto. Ao final da aplicação, os pesquisadores dirigiam os agradecimentos de praxe à turma, professores e diretores pela colaboração com a pesquisa.

\section{RESULTADOS}

Inicialmente, apresentam-se os resultados relativos às questões de pesquisa so bre (1) vantagens e desvantagens do O rkut, em termos de frequências e percentuais. Sequencialmente expõe-se (2) as análises da medida para aferir atitudes frente ao Orkut, que consistiu em Análise Fatorial (PAF; Principal A xis Factoring) e análise de consistência interna através do á e Análise Fatorial Confirmatória
(CFA; AMOS 7). Em seguida, (3) os resultados com relação às variáveis sócio-demográficas de acordo com os Testes t de Student, Análise de Variância de um fator (ANOVA oneway) e análises de Correlação de Pearson. Todas essas análises, excetuando-se a CFA, foram efetuadas mediante 0 programa estatístico SPSS na versão 15.

\section{Vantagens e desvantagens da participação no 0 rkut}

Com o intuito de conhecer melhor a motivação da participação no Orkut, buscou-se apreender as principais vantagens e desvantagens da participação neste site. Com base nas respostas dos participantes desse estudo foram construídas sete categorias considerando as respostas mais frequentes, como se observa na Tabela 1 abaixo.

TABELA 1 - Freqüência e percentual de vantagens e desvantagens da participação no Orkut

\begin{tabular}{|c|c|c|c|c|c|}
\hline Vantagens & $\mathbf{F}$ & $\%$ & Desvantagens & $\mathbf{F}$ & $\%$ \\
\hline $\begin{array}{l}\text { Fazer } \\
\text { amigos / } \\
\text { conhecer } \\
\text { pessoas }\end{array}$ & 158 & 35,6 & $\begin{array}{l}\text { Falta de } \\
\text { privacidade }\end{array}$ & 177 & 39,8 \\
\hline Comunicação & 100 & 22,5 & Vício & 38 & 8,5 \\
\hline Nenhuma & 17 & 3,8 & Nenhuma & 25 & 5,6 \\
\hline Bisbilhotar & 13 & 2,9 & $\begin{array}{l}\text { D ifamação / } \\
\text { Preconceitos }\end{array}$ & 22 & 4,9 \\
\hline $\begin{array}{l}\text { Entreteni- } \\
\text { mento }\end{array}$ & 7 & 1,6 & Perder tempo & 11 & 2,5 \\
\hline $\begin{array}{l}\text { Conheci- } \\
\text { mento }\end{array}$ & 6 & 1,3 & $\begin{array}{l}\text { Conhecer } \\
\text { pessoas } \\
\text { indesejáveis }\end{array}$ & 10 & 2,2 \\
\hline Convívio & 2 & 0,4 & G astar dinheiro & 10 & 2,2 \\
\hline Total & 303 & 100 & Total & 282 & 100 \\
\hline
\end{tabular}

D e acordo com a grande maioria das respostas dos participantes, pôde-se verificar que eles consideram "fazer amigos e conhecer pessoas" 
a maior vantagem da participação no Orkut. Por outro lado, consideram a "falta de privacidade" a maior desvantagem. Ainda foram elencadas como vantagens a "comunicação", "bisbilhotar", invadir a privacidade alheia, 0 "entretenimento" que proporciona o site e outras respostas não tão frequentes. As respostas de desvantagens ainda incluem o "vício", a dependência que o O rkut gera, "difamação" / "preconceitos", "perder tempo".

\section{Estrutura fatorial e consistência interna das atitudes frente ao 0 rkut}

Para se obter a estrutura fatorial (validade) dessa medida, foram inicialmente inspecionados 0 índice KMO e o Teste de esfericidade de Bartlett. O s resultados mostram um $\mathrm{KMO}=0,78$ e Teste de Esfericidade, $c^{2}=586,145$, estatisticamente significativo ( $p<0,001)$. Esses resultados indicam que a análise fatorial é adequada para os dados (Tabachnick \& Fidell, 2001).

Para retenção do item no fator, o item deveria ter carga fatorial maior ou igual a 0,30 e 0 fator eraconsiderado quando apresentasseeigenvalue (valor próprio) maior ou igual a 1. Como se esperava uma estrutura unifatorial, não se utilizou nenhuma rotação e não se fixou número de fatores a se extrair.

TABELA 2 - Estrutura fatorial e consistência interna das atitudes frente ao Orkut

\begin{tabular}{lccc}
\hline Itens & Cargas fatoriais & \multicolumn{2}{c}{ Comunalidades } \\
\hline AT 01 & 0,72 & 0,52 & 7,13 \\
AT 02 & 0,86 & 0,74 & 7,49 \\
AT 03 & 0,81 & 0,65 & 7,43 \\
AT 04 & 0,80 & 0,64 & 7,23 \\
& & & \\
Eigenvalue & & 2,51 & \\
\% variância & & $63,77 \%$ & \\
$\alpha$ de Cronbach & & 0,81 & \\
\hline
\end{tabular}

De acordo com a análise fatorial pelo método dos eixos principais (PAF), na Tabela 2 verificou-se que todos os itens da EAFO saturaram fortemente em um único fator (cargas fatoriais de 0,72 a 0,86 ) com valor próprio $>2$, com mais da metade da variância explicada por esse fator. A análise da consistência interna (precisão) mostrou-se igualmente favorável $(\mathrm{a}=0,81)$. Pelas médias nos 4 itens (média da pontuação total na escala $=7,32$ ), pode-se verificar que os participantes deste estudo mostram atitudes mais favoráveis frente ao site de relacionamento O rkut. Essaestruturaunidimensional foi corroborada através da CFA, que é uma técnica estatística para a validação de testes psicológicos (Pasquali, 2003) superior à análise fatorial tradicional e de componentes principais (Pasquali, 2003; Tabachnick \& Fidell, 2001). Através da CFA, obtiveram-se inicialmente os seguintes índices de ajuste da qualidade do modelo: $\mathrm{C}^{2} / \mathrm{gl}=6,150 ; \mathrm{GFI}=$ 0,99; $\mathrm{AGFI}=0,93 ; \mathrm{CFI}=0,99 ; \mathrm{RMR}=0,08$. Porém, por razões estatísticas, ao correlacionar-se os erros E1 eE3 (ver Figura 1), verificou-se um ajustemelhor: $\mathrm{c}^{2} / \mathrm{gl}=3,097 ; \mathrm{GFI}=1 ; \mathrm{AGFI}=0,96 ; \mathrm{CFI}=1 ; \mathrm{RMR}$ $=0,03$, pois os valores de $c^{2} / \mathrm{gl}$ estão próximos de 3 , os GFI,AG FI eCFI estão acima de 0,90 eo RMR está entre 0,05 e 0,08 (Byrne, 2001; Joreskög \& Sörbom, 1989; Tabachnick \& Fidell, 2001).

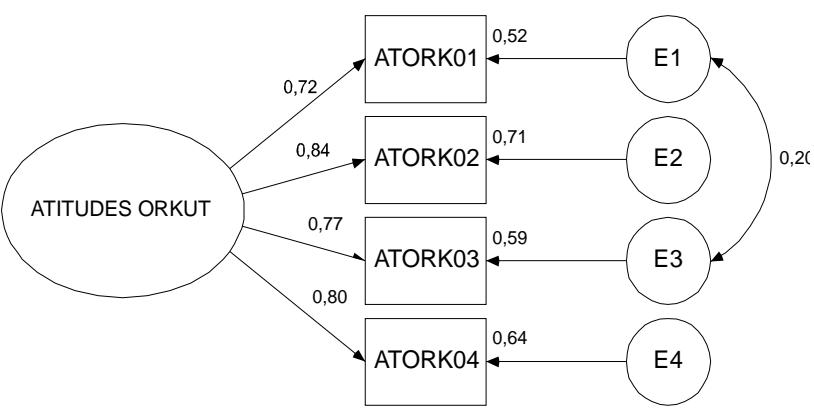

FIGURA 1 - Estrutura fatorial e parâmetros padronizados estimados da EAFO

\section{Atitudes frente ao 0 rkut e variáveis sócio-demográficas}

N esta parte apresentam-se os resultados relativos às comparações por grupos, formados com base nas variáveis sócio-demográficas, em relação a possibilidades de diferenças nas atitudes frente ao Orkut.

Foram considerados grupos formados por sexo, idade, tipo de escola, série e classe social. Através do Teste $t$ de comparação de médias para amostras independentes, na Tabela 3 é possível verificar diferenças estatisticamente significativas por grupo de idade e tipo de escola nas atitudes frente ao Orkut. A despeito das mulheres apresentarem médias mais altas $(\mathrm{m}=7,33)$ nas atitudes frente ao O rkut do que os homens ( $\mathrm{m}=7,30)$, essa diferença não foi estatisticamente significativa. 
Os grupos de idade foram divididos em grupo de 14-16 anos e grupo de 17-19 anos, pois formaram a grande maioria da amostra $(91,4 \%)$. Alunos com idades de 14 a 16 anos obtiveram as médias mais altas $(\mathrm{m}=7,46)$, mostrando atitudes mais favoráveis do que os de 17 a 19 anos $(\mathrm{m}=7,23)$. Ademais, verificou-se que aqueles de escolas públicas mostraram atitudes mais favoráveis $(\mathrm{m}=7,45) \mathrm{em}$ comparação àqueles de escolas privadas $(m=7,19)$.

TABELA 3 - Teste $t$ para médias nas atitudes frente ao O rkut por idade e tipo de escola

\begin{tabular}{lllllll}
\hline Idade & $\mathrm{N}$ & Médias & $\mathrm{dp}$ & $\mathrm{t}$ & $\mathrm{df}$ & $\mathrm{p}$ \\
\hline 14-16 & 174 & 7,46 & 1,09 & 1,936 & 404 & 0,05 \\
$17-19$ & 232 & 7,23 & 1,25 & & & \\
\hline Escola & & & & & & \\
\hline Pública & 219 & 7,45 & 1,29 & $-2,220$ & 442 & 0,03 \\
Privada & 225 & 7,19 & 1,09 & & & \\
\hline
\end{tabular}

As médias na pontuação total na medida de atitudes frente ao Orkut mostram que os alunos de primeira série $(\mathrm{m}=7,54)$ apresentam as atitudes mais favoráveis ( $2^{\mathrm{a}}$ série: $\mathrm{m}=7,26$ e $3^{\mathrm{a}}$ série: $\mathrm{m}=$ 7,24). Entretanto, de acordo com a ANOVA oneway, não se pôde verificar efeitos estatisticamente significativos das variáveis série $(\mathrm{F}(2,440)=2,539$, $\mathrm{p}>0,05)$ e classe socioeconômica $(F(4,436)=$ $1,270, p>0,05)$ nas atitudes frente ao Orkut. Por fim, verificaram-se correlações estatisticamente significativas $(p<0,05)$ das atitudes frente ao Orkut com horas de uso dainternet $(r=0,12)$ e dessaúltima variável com a idade $(r=-0,15)$, indicando que, quanto mais horas de internet, mais atitudes favoráveis frente ao Orkut e quanto menos idade, mais horas de uso da internet. A classe socioeconômica percebida (baixa, médiabaixa, média, média alta e alta) também se relacionou positiva, estatística e significativamente com as horas de uso de internet $(r=0,21, p<0,001)$. 0 que significa dizer que quanto maior a classe socioeconômica percebida, maior o número de horas de uso da internet.

\section{DISCUSSÃO}

Com base nas análises dos resultados desta pesquisa, verificaram-se (a) vantagens e desvantagens da participação no Orkut, (b) uma forma adequada de aferição das atitudes frente ao O rkut e (c) relacões com variáveis sócio-demográficas, como sexo, idade e horas de uso da internet.

\section{Vantagens e desvantagens da participação no 0 rkut}

A grande maioria das respostas dos participantes do presente estudo aponta para 0 comportamento de "fazer amigos e conhecer pessoas" como amaiorvantagem (atitudefavorável) da participação no Orkut e para a "falta de privacidade" como sendo a maior desvantagem (atitude desfavorável). Essa vantagem já fora sugerida como sendo umadas principais dos milhões de usuários de sites de relacionamento social (SNS), como o MySpace (Pierce, 2006) e o Facebook (Ellison et al., 2007) e da internet como um todo (Skitka \& Sargis, 2006). É interessante destacar que, a despeito dos estudantes apontarem a falta de privacidade como maior desvantagem, reportam que bisbilhotar é uma vantagem da participação no O rkut. Podemos interpretar que as pessoas gostam de bisbilhotar os perfis alheios, mas não se sentem bem ao serem bisbilhotadas. É possível que a percepção dessa desvantagem esteja associada ao medo de que informações do perfil sejam utilizadas com objetivos criminais.

Ainda tendo em análise os resultados sobre vantagens e desvantagens do 0 rk ut, chama a atenção um problema amplamente estudado em psicologia social, que se tratado preconceito. Como observado, os usuários deste site parecem sofrer também com esse problema, o que mostra ter sido providencial a iniciativa da $\mathrm{ONU}$ para combate do racismo, xenofobia e intolerância na internet (O NU Brasil, 2007). Nessa catego ria de resposta ainda se encontra a difamação. D epreende-se isso se tratar do que os especialistas convencionaram nomear como cyberbulling, o bulling (agressões, hostilidades) no espaço virtual, que está cada vez mais crescente e preocupantenas escolas (Li, 2006). O utro problema, desvantagem, que aparece nas respostas dos participantes é o vício, a dependência que gera o site de relacionamento Orkut. Esse tema se insere na compulsão com relação à internet, denominada por internet addiction, considerado um novo transtorno clínico (Young, 1996), muito prejudicial aos estudantes, ocasionando problemas acadêmicos (Young, 2004) e quadros de depressão (Y oung \& 
Rodgers, 1998). D estaque-se que esses problemas acadêmicos se relacionam com o tipo de resposta "perder tempo", que pode ser tido como o uso não racional da internet.

Em suma, as vantagens elencadas acima, como a de se comunicar e fazer amigos, certamente endossa a teoria de que o uso dos meios de comunicação, em particular da internet se prende à satisfação denecessidades específicas (Pierce, 2006).

\section{Análise psicométrica das atitudes frente ao 0 rkut}

De acordo com a teoria psicométrica clássica, o presente estudo conseguiu verificar que as atitudes frente ao Orkut podem ser mensuradas de modo válido, confiável (Anastasi \& Urbina, 2000; Pasquali, 2003) e breve, com o emprego de apenas 4 itens/ pares de adjetivos, numa escala tipo diferencial semântico (Crites et al., 1994) respondendo por mais da metade da variância explicada do construto. Através da técnica Alfa de Cronbach verificou-se que a escala é precisa. Ademais, os resultados da PAF indicam a unidimensionalidade da medida, corroborados com mais indicadores estatísticos na CFA (Byrne, 2001; Joreskög \& Sörbom, 1989; Tabachnick \& Fidell, 2001). Esses resultados oferecem apoio empírico a pesquisas realizadas em outros contextos que mostraram também a unidimesionalidade e precisão dos itens atitudinais propostos por Crites e cols. (Crites et al, 1994; Simons \& Carey, 2000), assim como aquelas realizadas no contexto nacional (Gouveia et al, 2005; G ouveia etal. 2007). Essa forma curta e prática de mensuração pode favorecer estudos em que se pretenda conhecer a influência de diversas, e mais complexas, variáveis nas atitudes em apreço, com outras escalas e mesmo na comparação entre culturas (G osling, Rentfrow \& Swann Jr., 2003) além de poder ser utilizada em contexto que não se possa contar com muito tempo, diminui as chances defadigae consequentes respostas dadas ao azar, sem a devida ponderação.

\section{Atitudes frente ao 0 rkut e variáveis sócio-demográficas}

No tocante às variáveis sóciodemográficas, pôde-se verificar que os estudantes de escolas públicas e privadas apresentaram atitudes favoráveis (medidas através da EAFO) frente ao site de relacionamento O rkut, independente de sexo, série e classe socioeconômica. Evidenciou-se, ainda, que os adolescentes mais jovens apresentaram atitudes mais favoráveis frente ao Orkut em comparação com os mais velhos, e os estudantes de escolas públicas apresentaram atitudes mais favoráveis em relação aos de escolas privadas. É possivel que os mais velhos e de escolas privadas também sejam mais críticos, apresentando por isso menos favorabilidade ao site.

Foi averiguado ainda que, quanto mais horas na internet eles relatavam dispender, mostravam também mais atitudes favoráveis frente ao O rkut e quanto menos idade, mais horas de uso da internet. A classe socioeconômica percebida também se relacionou positivamente com as horas de uso de internet. Esse dado é consoante com 0 levantamento realizado em todo o território nacional (IBGE, 2007). Obviamente, as classes mais favorecidas economicamente são as que têm maior facilidade na aquisição de computadores, acesso à internet e a todo o manancial de informações disponível. Nesse sentido, devem ser ampliados e intensificados os programas governamentais de inclusão digital, de barateamento de computadores e obtenção dessas máquinas por colégios públicos.

\section{CONSIDERAÇÕES FINAIS}

Em suma, podemos concluir que a maior contribuição da presente pesquisa consistiu em se conhecervantagens e desvantagens da participação no Orkut, uma proposta de mensuração das atitudes frente ao O rkut, de modo válido, preciso e rápido e conhecer algumas características relacionadas às atitudes frente ao Orkut. Estes e outros empreendimentos certamente serão importantes para melhor conhecer os sites de relacionamento social e suas funções sociais. Algumas sugestões de pesquisa podem ser destacadas, como a verificação da relação de traços de personalidade e bem-estar subjetivo com as atitudes frente ao Orkut, dos valores e comportamentos frente a este objeto social e das identidades sociais dos participantes das diversas comunidades que integram este site. 


\section{REFERÊNCIAS}

Anastasi, A., \& Urbina, S. (2000). Testagem psicológica. Porto Alegre: ArtMed.

Araújo, J. C. (2006). 0 que o meu aluno faz nesse tal de Orkut? Vida Educação. Fortaleza: Brasil Tropical, 3(9), 29-32.

Ajzen, I. (2001). Nature and operation of attitudes. Annual Review of Psychology, 52, 27-58.

Allport, G. W. (1966). Attitudes in the history of social psychology. In N. Warren \& M. Jahoda. (O rg.). Attitudes (pp. 15-21). London: Penguin Books.

Birnbaum, M. (2000). Psychological expeniments on the internet. New York: Academic Press. Recuperado em 10 jul. 2007, da Intro Web: http:/ / psych.fullerton.edu/ mbirnbaum/ web/ IntroWeb.htm

Byrne, B. M. (2001). Strutural equation modeling with amos: Basic concepts, applications, and programming. London: Lawrence Erlbaum Associates, Publishers.

Byrne, D. (1971). The attraction paradigm. New York: Academic Press.

Chen, Q., \& Wells, W. D. (1999). Attitude toward the site. Journal of Advertising Research, 39(5), 27-37.

Crites S. L., Fabrigar, L. R., \& Petty, R. E. (1994). Measuring the affective and cognitive properties of attitudes: Conceptual and methodological issues. Personality and Social Psychology Bulletin, 20(6), 619-34.

Ellison, N. B., Steinfield, C., \& Lampe, C. (2007). The benefits of Facebook "friends:" Social capital and college students' use of online social network sites. Journal of ComputerMediated Communication, 12(4), Recuperada em 10 jul. 2007, da Ellison: http:/ / jcmc.indiana.edu/ vol12/ issue4/ ellison.html

Fischoff, S. (2005). Media psychology: A personal essay in definition and purview. Journal of Media Psychology, 10(1). Recuperada em 10 jul. 2007, da Journal of Media Psychology: http:/ / www.calstatela.edu/ faculty/ sfischo
Giles, D. (2003). Media psychology. New Jersey: Lawrence Erlbaum Associates, Publishers.

Gouveia, V. V., Pimentel, C. E., Medeiros, E. D., G ouveia, R. S., \& Palmeira, J. N. (2007). Medindo atitudes frente ao uso de drogas: Evidências de validade fatorial e preditiva de uma escala. Jornal Brasileiro de Psiquiatria, 56(1), 53-59.

Gouveia, V. V., Pimentel, C. E., Queiroga, F., Meira, M., \& Jesus, G. R. (2005). Escala de atitudes frente ao uso de maconha: Comprovação da sua validade de construto. Jornal Brasileiro de Psiquiatria, 54(1), 5-12.

G osling, S. D., Rentfrow, P. J., \& Swann Jr., W. B. (2003). A very brief measure of the big-five personality domains. Journal of Research in Personality, 37, 504-528.

G osling, S. D., Vazire, S., Srivastava, S., \& John, O. P. (2004). Should we trust Web-based studies? A comparative analysis of six preconceptions about Internet questionnaires. American Psychologist, 59, 93-104.

Hamann, F. P. (2004). Comunidade orkutiana brasileira: Considerações sobre os jovens e o orkut no Brasil. Trabalho de Conclusão de Curso. Pontifícia Universidade Católica do Rio de Janeiro - PUC-RJ, D epartamento de Psicologia.

Joresköb, K., \& Sörbom, D. (1989). Lisrel 7 user's reference guide. Mooresville: Scientific Software.

Kraut, R., Patterson, M., Lundmark, V., Kiesler, S., Mukopahyay, T., \& Scherlis, W. (1998). Internet paradox: A social technology that reduces social involvement and psychological well-being? American Psychologist, 53(9), 1017-1031.

Lee, B. K., Hong, J. Y., \& Lee, W. N. (2004). How attitude toward the web site influences consumer brand choice and confidence while shopping online. Journal of ComputerMediated Communication, 9(2). Retirado em 03 out. 2007, da Published by The Annenberg School at USC: http:/ / jcmc.indiana.edu/ vol9/ issue2/ lee.html 
Li, Q. (2006). Cyberbullying in schools: A research of gender differences. School Psychology International, 27(2), 157-170.

Luskin, B. J. (2003). Media psychology: A field whose time is here. The California Psychologist, 15.

McKenna, K. Y. A., \& Bargh, J. A. (2000). Plan 9 from cyberspace: The implications of the Internet for person-ality and social psychology. Personality and Social Psychology Review, 4(1), 57-75.

McMillan, S. J., Hwang, J. S. \& Lee, G. (2003). Effects of structural and perceptual factors on attitudes toward the website. Journal of Advertising Research, 43(4), 400-409.

Media Psychology (2007). Division 46 of American Psychological Association. Recuperado em 05 set. 2007, da American Psychological Association: http:/ / www.apa.org/ divisions/ div46/

O liveira, M. E. (2007). Orkut: 0 impacto da realidade da infidelidade virtual. D issertação de Mestrado em Psicologia. Pontifícia Universidade Católica do Rio de Janeiro.

ONU Brasil (2007). Centro de notícias ONU. Expertos de la ONU examinan racismo en internet. Recuperado em 19 set. 2007 do Centro de Noticias ONU: http:/ / w w w. un.org/spanish/ N e w s/ fullstorynews.asp?newsI $D=6107 \&$ criteria1 $=$ racismo $\&$ criteria $2=$ internet .

ONU Brasil (2007). Centro de notícias ONU. Relator especial subraya urgencia de legislación contra pornografía infantil en Internet. Recuperado em 19 set. 2007 do Centro de Noticias ONU: http:/ / ww w. un.org/spanish/ N e w s / fullstorynews.asp?newsI D $=4351 \&$ criteria1 $=$ niños\& criteria2

Pasquali, L. (2003). Psicometria: Teoria dos testes na psicologia e na educação. Petrópolis, RJ: Vozes.

Pereira, M. E., Silva, J. F., \& Silva, P. B. (2006). Investigações psicológicas no ciberespaço: 0 impacto do interesse, filiação grupal e conhecimento na adesão às crenças ufológicas. Interação em Psicologia, 10(2), 375-384.
Pierce, T. A. (2006). X-Posed on MySpace: A content analysis of "MySpace" social networking sites. Journal of Media Psychology, 12(1). O nline Publication D ate: February 10, 2007. Recuperado em 10 jul. 2007, do Centro de Noticias ONU: http:/ / www.calstatela.edu/ faculty/ sfischo/

Pierce, T. A. (2006).Talking to strangers on MySpace: Teens' use of internet social Networking Sites. Journal of Media Psychology, 11(3). O nline Publication D ate: November 7, 2006. Recuperado em 10 jul. 2007, do Centro de Noticias O NU: http:/ / www.calstatela.edu/ faculty/ sfischo/

Rheingold, H. (1993). The virtual community: Homesteading on the electronic frontier. Reading, Massachusetts: Addison-Wesley.

Rodrigues, A., Assmar, E. M. L., \& Jablonski, B. (2000). Psicologia social. Petrópolis, RJ: Vozes.

Shergill, G. S., \& Chen, Z. (2005). Web-based shopping: Consumers' attitudes towards online Shopping in New Zealand. Journal of Electronic Commence Research, 6(2), 79-94.

Simons, J., \& Carey, K. B. (2000). Attitudes toward marijuana use and drug-free experience: Relationships with behavior. Addictive Behaviors, 25(3), 323-31.

Smith, E. R. \& Mackie, D. M. (1995). Social psychology. New York: Worth Publishers.

Stoll, C. (1995). Silicon snake oil: Second thoughts on the information highway. New York: D oubleday.

Skitka, L. J., \& Sargis. E. G. (2006). The internet as psychological laboratory. Annual Review of Psychology, 57, 529-555.

Tabachnick, B. G. \& Fidell, L. S. (2001). Using multivariate statistics. New York: HarperCollins.

Teske, J. A. (2002). Cyberpsychology, human relationships, and our virtual interiors. Zygon ${ }^{\circledR}, 37(3), 677-700$.

Tice, D. M., Butler J. L., Muraven M. B., \& Stillwell A. M. (1995). When modesty prevails: Differential favorability of self-presentation to friends and strangers. Journal of Personality and Social Psychology, 69, 1120-1138. 
Turkle, S. (1995). Life on the screen: Identity in the age of the internet. New York: Simon $\&$ Schuster.

Wallace, P. (1999). The psychology of internet. Cambridge: Cambridge University Press.

Wu, G. (1999). Perceived interactivity and attitude toward website. In Annual Conference of the American Academy of Advertising, Albuquerque, New Mexico.

Young, K. S. \& Rodgers, R. C. (1998). The relationship between depression and internet addiction. CyberPsychology \& Behavior, 1(1), 25-28.

Young, K. S. (1996). Internet addiction: The emergence of a new clinical disorder. In Paper presented at the 104 annual meeting of the American Psycho-logical Association. Toronto: American Psychological Association.

Young, K. S. (2004). Internet addiction: A new clinical phenomenon and its consequences. American Behavioral Scientist, 48(4), 402-415.

Zajonc, R. (1968). Cognitive theories in social psychology. In G. Lindzey \& E. Aronson (O rg.). The handbook of social psychology (pp. 320-411). California: Addison-Wesley.

Recebido: 18/ 04/ 2008

Received: 04/ 18/ 2008

Aprovado: 02/ 07/ 2008

A pproved: 07/ 02/ 2008 